J. Lake Sci. (湖泊科学), $2007,19(1): 18-24$

http:// www. jlakes. org. E-mail: jlakes@ niglas. ac.cn

(c) 2007 by Journal of Lake Sciences

\title{
太湖底泥和疏竣堆场中持久性有机污染物的分布及潜在生态风险
}

\author{
张 路 ${ }^{1}$, 范成新 ${ }^{1}$, 鲜启鸣 ${ }^{2}$, 马莹莹 ${ }^{2}$, 赵中华 ${ }^{2}$, 邹惠仙 ${ }^{2}$ \\ (1: 中国科学院南京地理与湖泊研究所, 南京 210008) \\ (2: 南京大学环境学院, 南京 210093)
}

摘 要: 以太湖的重污染湖区-五里湖为例, 以有机氯农药和多环芳烃为主要目标化合物, 分析了疏竣湖区底泥中典型 持久性有机污染物的蓄积规律和对生态的潜在影响. 分析结果表明:五里湖疏浚湖区底泥中的多环芳烃类有机物在深度 上呈指数分布特征,上层底泥中的含量显著高于下层,但对湖区生态没有显著影响;五里湖底泥受到一定的有机氯农药的 污染, 以六六六和 DDE 等污染为主, 但对比生态风险评估指标仍属于生态安全的底泥. 疏竣堆场土壤中多环芳烃的含量 仅为五里湖疏竣底泥的 $1 / 6$, 在堆场使用后 8 个月后,堆场底泥中 16 种优先检测的多环芳烃含量与湖区底泥仍相当. 但 堆场使用后, 堆场的水花生皘秆内多环芳烃含量发生了显著变化,其总含量增加为原来的 6 倍,有四种多环芳烃含量超过 了生态风险效应区间低值但低于效应区间高值. 原秥秆内不含有的苯并 $[a]$ 葱和营也被检出, 说明堆放五里湖疏竣底泥 后对植物产生了一定的生态威胁.

关键词:有机氯农药;多环芳烃;底泥; 疏竣;生态风险;太湖

\section{Persistent Organic Pollutants (POPs) distribution and potential ecological risk on sediment in Lake Taihu and slurry stockyard}

\author{
ZHANG Lu ${ }^{1}$,FAN Chengxin ${ }^{1}$,XIAN Qiming ${ }^{2}$, MA Yingying ${ }^{2}$, ZHAO Zhonghua $^{2} \&$ ZHOU Huixian $^{2}$ \\ (1:Nanjing Institute of Geography and Limnology, CAS, Nanjing 210008, P. R. China) \\ (2:Environmental School of Nanjing University, Nanjing 210093,P. R. China)
}

\begin{abstract}
As an example of severe polluted lake zone in Lake Taihu, the organochlorine pesticide and polycyclic aromatic hydrycarbons (PAHs) in sediment in Wuli Lake was analyzed to determine the accumulation regulation in sediment and potential ecological risk to aquatic system and slurry stockyard after dredging. The results showed that the PAHs in sediment in Wuli Lake were exponentially distributed along the depth and the contents in upper layer were remarkably higher than in the lower layer, but no obvious ecological impact was found. The sediment in Wuli Lake was polluted by organochlorine pesticide in some what degree, especially for hexachloro cyclohexane ( $\mathrm{HCH}$ ) and DDE, but they were possibly safe to aquatic system. The PAHs in soil in slurry stockyard was only one sixth of the PAHs in sediment in Wuli Lake. Eight months after the stockyard was used, the 16 priority PAHs in sediment in the stockyard was still comparable to the original lake sediment, but the contents of PAHs in the aquatic plant (alligator alternanthera) remarkably changed to six times of the original contents before the use of stockyard and the contents of four species of the PAHs were exceeded the ERL but lower than the ERM. The Benzo[a] anthracene and Chrysene was also detected in the plant stem which was not detected before the use of stockyard. This showed the sediment in the stockyard from the lake dredging would put some threaten on the ecological safety of water plant in the stockyard.
\end{abstract}

Keywords: Organochlorine pesticide; polycyclic aromatic hydrycarbons (PAHs); sediment, dredging, ecological risk; Lake Taihu

* 国家“十五”重大科技专项(863) 项目(2002AA601013), 基础研究重大项目前期研究专项(2001CCB00700)和国家 自然科学基金 $(40501064,20577053$ )联合资助. 2006-02-13 收稿;2006-09-13 收修改稿. 张路,男, 1975 年 生, 博士, 助理研究员; E-mail: luzhang@ niglas. ac. cn. 
疏竣作为一种去除持久性化学污染物的湖泊治理方法已经被广泛运用于世界各地的水环境治理工程 中. 通过疏竣, 湖泊底泥中保守性的污染物质, 如多环芳烃 (PAHs)、多氯联苯 (PCBs)、有机氯农药、重金属 $(\mathrm{Pb}, \mathrm{Cu}, \mathrm{Cr})$ 等被移出湖体, 从而减轻这些具有毒性或者潜在毒性的物质对湖泊水体和底泥中微生物、小型 生物以及具有较高营养级的大型水生动物的毒性威胁. 许多国家采用疏竣的方式来减轻底泥中有机毒性 物质和重金属对底栖生物的毒性作用,并达到改善水环境质量的目的,并开展了疏竣对有机毒性物质的生 态效应研究, 如美国 Hudson 河在受 GE 公司影响, 发生严重的 PCBs 污染, 导致底泥中底栖微生物数量的减 少, 并间接导致水体中鱼类大量减少,而且鱼类体内 PCBs 含量大大超过美国环保局 (EPA) 和食物药品管理 局 (FDA) 公布的食用标准. 因此, 在上世纪后半页, 旨在去除底泥中保守性 PCBs 污染物的疏竣工程在 Hudson 河大规模展开 ${ }^{[1]}$, 约有 40 英里 $(64 \mathrm{~km})$ 长的河道被彻底疏竣. 类似的研究在埃及的苏伊士运河 ${ }^{[2]}$ 和荷兰的 Delft 湾 ${ }^{[3]}$ 也曾经开展过.

我国的许多湖泊都曾经或者正在进行底泥疏竣,但有关疏竣及疏竣底泥对堆场的生态风险评价研究则 开展的甚少. Long ${ }^{[4-7]}$ 最早开始对水环境中底泥的多环芳烃、农药、PCBs 等持久性有毒有机污染物进行生 态风险的效应研究. 美国国家环保局 $(\mathrm{EPA})$ 和美国国家海洋大气局 (NOAA) ${ }^{[8]}$ 等组织在 Long 等人的研究 基础上分别制定了针对 PAHs、PCBs 等持久性有机污染毒性物质以及重金属类物质的含量标准,用以评估 底泥和土壤中这些物质的含量是否可能造成环境威胁和是否对周围生物和微生物造成立即或者潜在的毒 性威胁,作为判断疏竣工程是否成功,疏竣底泥的处置是否适当的标准. 虽然不同水域底泥和底泥堆场的 持久性有机污染毒性物质的本底不同,但依据环境生物毒理学评价得到的有机污染物的潜在生态风险的效 应区间低值 (effects range low, ERL, 生物有害效应几率 $<10 \%$ ) 和效应区间中值 (effects range median, $\mathrm{ERM}$,生物有害效应几率 $>50 \%$ ), 则具有广泛意义上的共性, ERL 和 ERM 因此又被视为底泥的生态风险 标志水平 (guidelines), 具有普适意义.

本文结合太湖五里湖底泥的疏竣工程措施, 对湖区疏竣底泥中的持久性有机污染物以及疏竣后底泥在 堆场的分布和潜在生态风险进行初步研究. 由于准确的生态风险评价必须考虑受体,暴露和效应等科学问 题, 而不能仅凭沉积物的沉积物环境质量基准进行定量评价, 本文仅从湖泊疏竣及其后效方面进行疏竣的 生态效应进行尝试.

\section{1 研究区域和采样点}

五里湖位于太湖东北部,为半封闭型城市湖泊. 研究的疏竣区域为西五里湖 (图 1). 疏竣采用环保绞 吸式疏竣船进行, 疏竣底泥通过输泥管输送到位于鱼港乡的底泥堆场 (图 1). 堆场原为干化鱼塘, 堆场鱼 塘内原有水花生和其他农作物生长. 2003 年 11 月,五里湖疏竣保留区开始进行疏竣后,堆场启用. 堆场使

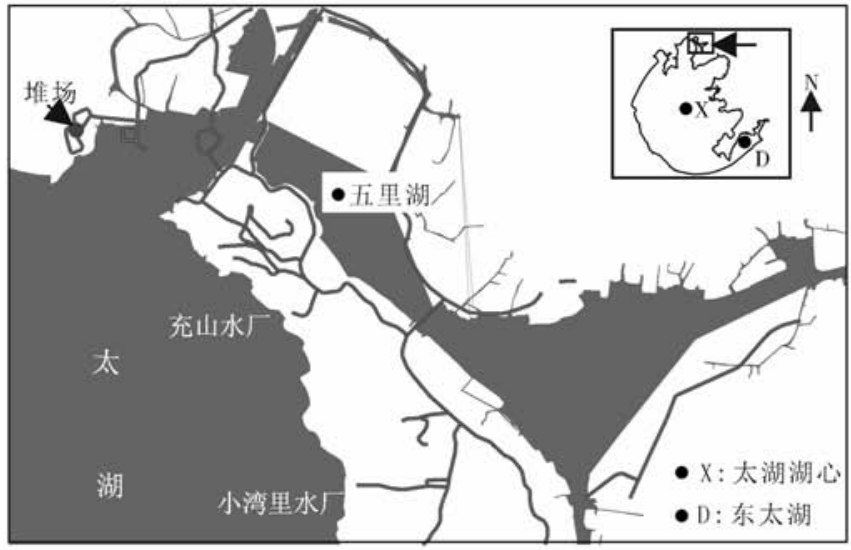

图 1 五里湖疏竣区域和底泥堆场示意图

Fig. 1 Sketch map of dredging location and slurry stockyard of Wuli Lake 
用后, 原有的水花生仍然能够继续生长, 而其他农作物则由于淹水无法生长. 因此, 本研究除了对疏竣区底 泥、堆场内底泥和堆场内原土壤进行采样外, 还选用了本地种水花生作为目标植物进行有机污染物分析. 采样点共 2 个. 其中在五里湖样点用无扰动柱状采样器采集了疏竣前底泥柱样 3 个 $(25 \mathrm{~cm}$ 深, 共分五层, 每层 $5 \mathrm{~cm}$ ), 作为对照, 还用 Peterson 采泥器采集了太湖湖心 $(X)$ 和东太湖 $(D)$ 各一个表层底泥样进行有机 氯农药的比较研究. 在堆场样点,分两次 (堆场使用前和使用后) 分别采集了使用前堆场土壤和使用后堆场 底泥, 同时在采样点附近挑选茎长和茎粗相近的植物, 采集时, 从植物根部开始计算, 采集等长度 (约 $40 \mathrm{~cm}$ 长) 的样品.

\section{2 研究方法}

疏竣生态效应研究分为两类:西五里湖受浚底泥和堆场底泥、水花生. 利用气相色谱 - 质谱联用技术 (GC-MS) 对受浚底泥中主要有机污染物进行了定性分析, 定量分析了其中的有机氯农药和多环芳烃 $(\mathrm{PAHs})$ 类物质. 对于堆场底泥和水花生则定性定量分析了其中的 PAHs 类物质. 其中, 底泥和水花生按如 下方法进行分析:

底泥经剔除杂质和植物残余物后, 以四分法取出约 $20 \mathrm{~g}$, 经冷冻干燥机中干燥 $48-72 \mathrm{~h}$ 后用研钵研磨 过 100 目篮后保存待用. 植物样品亦经洗净后冷冻干燥,并粉碎后待用.

(1) PAHs: 准确称取干燥的 5-20 g 底泥或植物样品于三角烧瓶中, 同时定量加人 $50 \mathrm{ml}$ 二氮甲烷, 超 声萃取 $1 \mathrm{~h}$. 离心后移取上层萃取清液, 重复一次萃取, 样品再用 $10 \mathrm{ml}$ 二氯甲烷洗涤, 合并萃取液于梨形瓶 内. 加人适量无水 $\mathrm{Na}_{2} \mathrm{SO}_{4}$ 干燥后旋转蒸发至 $2.0 \mathrm{ml}$. 采用硅胶柱层析法进行样品纯化. 以石油醚洗脱烷 烃, 二氯甲烷洗脱 PAHs. 洗脱液收集人 $25 \mathrm{ml}$ 梨形瓶中, 用无水硫酸钠干燥后用旋转蒸发仪浓缩至 $1 \mathrm{ml}$ 左 右,再用 $\mathrm{N}_{2}$ 缓缓吹扫溶剂至 $0.1 \mathrm{ml}$.

(2) 有机氯农药: 准确称取干燥的 $5 \mathrm{~g}$ 底泥样品于 $100 \mathrm{ml}$ 离心管中, 同时定量加人 $4 \mathrm{ml}$ 重蒸水, $10 \mathrm{ml}$ 甲醇及 $20 \mathrm{ml}$ 正己烷, 密封后超声萃取 $1 \mathrm{~h}$. 离心后移取上层萃取清液,再加人 $20 \mathrm{ml}$ 正已烷, 再萃取一次, 合 并萃取液并加人足量无水 $\mathrm{Na}_{2} \mathrm{SO}_{4}$ 干燥, 将萃取液浓缩至 $2.0 \mathrm{ml}$. 采用硅胶柱层析净化样品. 准确称取 1.5 $\mathrm{g}$ 硅胶和 $0.5 \mathrm{gNa}_{2} \mathrm{SO}_{4}$ 至 $6 \mathrm{ml}$ 固相萃取柱, 萃取柱用 $10 \mathrm{ml}$ 正已烷清洗两次, 将萃取浓缩的样品溶液加于萃 取柱顶部, 用 $10 \mathrm{ml} 5 \%$ 异丙醇/正己烷溶液洗脱,洗脱液用旋转蒸发仪浓缩至 $1 \mathrm{ml}$ 左右后用 $\mathrm{N}_{2}$ 缓缓吹扫溶 剂至 $0.1 \mathrm{ml}$.

定性分析通过计算机标准 NIST 质谱库检索进行,并结合标准化合物的保留时间比较和计算 Lee 保留 指数对比进行. 定量分析采用内标法结合选择离子峰面积 (SIM) 进行. 色谱条件: DB - 5MS 毛细管柱 (30 $\mathrm{m} \times 0.25 \mathrm{~mm} \times 0.25 \mu \mathrm{m}$, 美国 J \& W 公司), 流速: $1 \mathrm{ml} / \mathrm{min}$; 升温程序: $40^{\circ} \mathrm{C}$ 保持 $5 \mathrm{~min}$, 以 $8^{\circ} \mathrm{C} / \mathrm{min}$ 的速率上 升到 $280^{\circ} \mathrm{C}$, 保持 $11 \mathrm{~min}$, 不分流进样,进样量: $1 \mu \mathrm{l}$. 质谱条件: 离子源能量 $70 \mathrm{eV}$; 进样口温度: $250^{\circ} \mathrm{C}$; 质量扫 描范围:45-450 amu. 样品分析前, 采用选择性离子法对 EPA625 混标标样进行检测, 各测定 6 次. 各化合 物的精密度在 $2 \%-15 \%$. 配制不同浓度的标样,进行分析, 所得的标准曲线的线性关系良好, 相关系数 $r$ 均达到 0.99 , 以仪器三倍噪声所对应的浓度作为检测限, 低于检测限的样品看作未检出 (nd).

\section{3 结果和讨论}

\section{1 五里湖疏竣区底泥有机毒性物质污染历史变化和疏竣风险}

3.1 .1 定性评价 GC/MS 定性分析结果显示(表 1), 五里湖受浚湖区底泥中共检出有机物近 70 种, 其中具 有生物毒性或潜在生物毒性化合物 20 多种(不包括其同系物), 部分优先污染物也在五里湖疏竣底泥中被 检出, 如农药类的 DDT 及其衍生物、六六六及其同分异构体、16 种 EPA 优先检测多环芳烃( 指母体化合物, 不包括其衍生物和同系物) 中有 13 种优先检测化合物被不同程度的检出,而其同系物 (即各种取代化合 物) 的种类更多.

从表 1 可以看出,五里湖疏竣区域底泥中有机毒性物质种类主要为取代苯类 (21 种)、有机酯类 (6 种)、多环芳烃类物质 (13 种)、农药类 (包括有机氯和有机磷农药 18 种) 以及其他有机污染物 (5 种). 五里 湖疏竣区域底泥中毒性有机物的种类比较丰富,这么多被检出的毒性有机物的存在可能对水体产生一定程 
度的生态影响.

表 1 五里湖疏竣区底泥主要检出微量有机物

Tab. 1 Qualified trace organics in sediments of dredged area in Wuli Lake

\begin{tabular}{|c|c|c|c|c|c|c|c|}
\hline 类别 & \multicolumn{7}{|c|}{ 有机物名称 } \\
\hline 多环芳烃类 & 菲 & 葸 & 范 & 茭 & 苂葸 & 芘 & 芴 \\
\hline \multirow{4}{*}{ 取代苯类 } & 苯并 (a) 芘 & 苯并 (a)蒽 & 苯并 (b) 苂蒽 & 营 & $\begin{array}{c}\text { 狮并 }(1,2,3 \\
- \text { cd) 芘 }\end{array}$ & $\begin{array}{c}\text { 苯并 } \\
(\mathrm{g}, \mathrm{h}, \mathrm{i}) \text { 芘 }\end{array}$ & \\
\hline & 间-二氯苯 & 对-二氯苯 & 邻-二氯苯 & 硝基苯 & 邻硝基苯酚 & $\begin{array}{l}\text { 二(2-氯异 } \\
\text { 丙基)醚 }\end{array}$ & 2,4 -二甲酚 \\
\hline & 六氯乙烷 & 2,4-二氯酚 & $\begin{array}{c}2,4,6 \\
\text {-三氯酚 }\end{array}$ & $\begin{array}{c}1,2,4 \\
\text {-三氯苯 }\end{array}$ & 五氯酚 & 六氯苯 & 2-氯酚 \\
\hline & 2-氯萘 & 对硝基酚 & 4-氯苯基苯醚 & 偶氮苯 & $\begin{array}{l}\text { 2-甲基-4,6 } \\
\text {-二硝基酚 }\end{array}$ & $\begin{array}{c}2,6 \text {-二硝 } \\
\text { 基甲苯 }\end{array}$ & $\begin{array}{c}2,4-二 \text { 二硝 } \\
\text { 基甲苯 }\end{array}$ \\
\hline 邻苯二甲 & 邻苯二甲酸 & 邻苯二甲酸 & 邻苯二甲酸二 & 邻苯二甲酸 & 邻苯二甲酸 & 邻苯二甲酸 & \\
\hline 酸酯类 & 二丁酯 & 丁基苯基酯( & (2-乙基已基)酯 & 旨 二辛酯 & 二乙酯 & 二甲酯 & \\
\hline 农药类 & $\begin{array}{c}\alpha-\text { 六六六 } \\
\text { 七氮 } \\
\text { 治蛽磷 }\end{array}$ & $\begin{array}{c}\beta \text {-六六六 } \\
\text { 艾氏剂 } \\
\text { 乙拌磷 }\end{array}$ & $\begin{array}{c}\gamma \text {-六六六 } \\
\alpha \text {-硫丹 } \\
\text { 乙基对硫磷 }\end{array}$ & $\begin{array}{c}\text { 林丹 } \\
\beta-\text { 硫丹 } \\
\text { 甲基对硫磷 }\end{array}$ & $\begin{array}{c}\text { 4. 4' - DDE } \\
\text { 硫酸硫丹 }\end{array}$ & $\begin{array}{l}\text { 阿特拉津 } \\
\text { 异狄氏剂 }\end{array}$ & $\begin{array}{c}\text { 西玛津 } \\
\text { 异狄氏醛 }\end{array}$ \\
\hline 其他 & 异佛尔酮 & 六氯环戊二烯 & 六氯丁二烯 & $\begin{array}{c}4 \text { - 澳苯基 } \\
\text { 苯醚 }\end{array}$ & $\begin{array}{l}\text { 二(2 - 氯乙 } \\
\text { 氧基) 甲烷 }\end{array}$ & & \\
\hline
\end{tabular}

3.1.2 PAHs 定量结果五里湖疏竣区域底泥中 16 种优先检测多环 芳烃(实际在底泥柱样的不同深度中共检出 13 种)的总含量在底泥 中的垂向分布呈显著的指数分布特征 (图 2). 上层底泥含量显著高 于下层. 13 种优先检测 PAHs 在五里湖疏竣区域表层底泥中的含量 约为 $300 \mathrm{ng} / \mathrm{g}$, 而且随着深度的增加, 其底泥中的含量趋向更低. 就表 层底泥中 PAHs 含量而言, 与前期在太湖其他湖区 (大浦口和东太湖) 的底泥中测得的含量基本相当 ${ }^{[9,10]}$. 该含量与世界其他湖泊相比, 处 于中等水平. 说明与太湖的其他湖区和其他湖泊相比, 五里湖的多环 芳烃类污染并非十分突出. 但是, 由于底泥中 PAHs 含量呈指数分布, 且由于其保守性, 从这个意义上说, 疏竣能显著减少该类保守性微量 有机毒物在水环境中的蓄积,也能减轻 PAHs 对底泥中底栖微生物和 生物的毒害作用.

采用 ERL 和 ERM 值对底泥中 PAHs 对底栖微生物和生物的潜在 生态风险进行评价. 一般而言, 低分子量(2-3 环) PAHs 可呈现显 著的急性毒性, 而某些高分子量 PAHs 则具有潜在的致癌性. 本评估

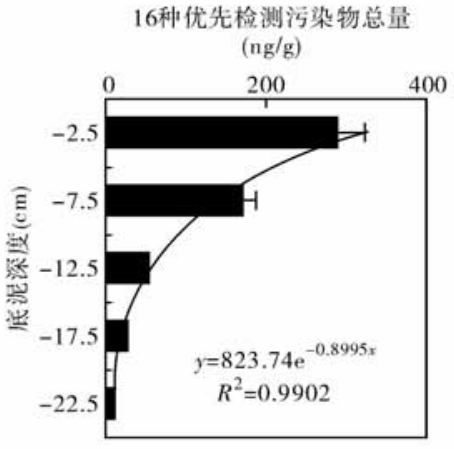

图 2 五里湖疏竣区 16 种优先检测 PAHs 总含量垂直分布

Fig. 2 Vertical Profile of contents of total16 priority PAHs in sediment of dredging area in Wuli Lake 将五里湖疏竣区域底泥中 9 种 $\mathrm{PAHs}$ 与相应的底泥生态风险标志水 平进行比较,结果表明(表 2): 除了表层 $10 \mathrm{~cm}$ 内芴的含量已处于 $\mathrm{ERL}$ 与 ERM 之间外,其他 PAHs 组分含 量均低于 ERL. 因此, 除了表层 $10 \mathrm{~cm}$ 内的芴可能对底泥内底栖生物产生一定的生态影响外, $10 \mathrm{~cm}$ 深度下 的底泥中多环芳烃的潜在生态风险则很小. 而从多环芳烃总量看, 五里湖疏竣区底泥的含量远远低于效应 区间低值(ERL).

\section{2 有机氯农药生态风险评价结果}

五里湖疏竣湖区底泥中有机氯农药主要监测出六六六 $(\mathrm{HCH}) 、$ DDE 两种. 其含量与太湖其他湖区 (湖 心区和东太湖) 相比略高 (表 3). 但是从底泥有机氯农药的潜在生态风险的效应区间值分析 (参考美国 NOAA 标准), 五里湖以及太湖的东太湖和湖心区域底泥中有机氯农药的含量均小于效应区间低值( ERL), 说明这些底泥中的农药含量尚不足以产生显著的生态风险. 但注意到五里湖底泥中三种六六六的异构体 
均有检出, 而其他两个湖区底泥中均未检出, 说明相对于东太湖和太湖湖心区, 五里湖底泥确实受到一定程 度的有机氯农药污染, 应引起重视.

表 2 五里湖不同深度底泥中 PAHs 组分的含量分布

Tab. 2 Contents of PAHs species in sediment in different depth in Wuli Lake

\begin{tabular}{|c|c|c|c|c|c|c|c|}
\hline \multirow{2}{*}{ PAHs } & \multirow{2}{*}{$\begin{array}{c}\text { ERL } \\
(\mathrm{ng} / \mathrm{g})\end{array}$} & \multirow{2}{*}{$\begin{array}{c}\text { ERM } \\
(\mathrm{ng} / \mathrm{g})\end{array}$} & \multicolumn{5}{|c|}{ PAHs 含量均值 $(\mathrm{ng} / \mathrm{g})$} \\
\hline & & & $0-5 \mathrm{~cm}$ & $5-10 \mathrm{~cm}$ & $10-15 \mathrm{~cm}$ & $15-20 \mathrm{~cm}$ & $20-25 \mathrm{~cm}$ \\
\hline 萗 & 160 & 2100 & 0.25 & 17.69 & 24.28 & 21.45 & 4.85 \\
\hline 芴 & 19 & 540 & 37.83 & 23.21 & 2.18 & 0.32 & 0.55 \\
\hline 菲 & 240 & 1500 & 59.48 & 32.3 & 0.42 & 0.08 & 0.09 \\
\hline 葸 & 85 & 1100 & 1.77 & 4.82 & 7.90 & 1.20 & 1.97 \\
\hline 苂葋 & 600 & 5100 & 69.25 & 31.46 & 3.12 & 0.33 & 0.57 \\
\hline 芘 & 665 & 2600 & 38.20 & 21.07 & 2.66 & 0.33 & 0.58 \\
\hline 䓛 & 384 & 2800 & 38.79 & 13.53 & 11.61 & nd & nd \\
\hline 苯并 (a) 蒽 & 261 & 1600 & 18.17 & 13.54 & nd & nd & nd \\
\hline 苯并 (a) 芘 & 430 & 1600 & 6.72 & 12.22 & nd & nd & nd \\
\hline$\sum \mathrm{PAHs}$ & 4022 & 44792 & 289.23 & 169.83 & 52.17 & 23.715 & 8.62 \\
\hline
\end{tabular}

\section{4 底泥堆场使用前后 $\mathrm{PAHs}$ 含量及潜在生态风险}

\section{1 堆场使用前后底泥 PAHs 生态风险变化}

堆场原土壤中 PAHs 总含量仅为五里湖疏竣区表层底泥 PAHs 含量的约 $1 / 6$, 为 $48 \mathrm{ppb}$. 各 PAHs 组分 均明显低于 ERL 值 (表 4). 堆场泥样堆积 8 个月后, 16 种优先检测多环芳烃总量 (241.7 ppb) 与疏竣湖区 表层底泥含量 $(289.2 \mathrm{ppb}$ )仍相当, 未显示明显降解趋势, 其各组分也未发生显著变化. 显示出该类化合物 的保守性,即持久性,且含量仍然低于 ERL 值.

\section{2 堆场使用前后 PAHs 在水生植物体内蓄积变化}

在底泥堆积前后,堆场内水生植物 (水花生)秸秆内的多环芳烃发生了显著变化. 堆场使用 8 个月后与 使用前相比,堆场内生长的水花生中主要优先检测多环芳烃总量增加约 6 倍. 所有单体多环芳烃均有不同 倍数的增加. 一些底泥堆积前植物体内未有检出的多环芳烃,如苯并 $[\mathrm{a}, \mathrm{h}]$ 葱和萤等,在堆场使用后被检出 (图 3), 证明这些物质来自于疏竣底泥的污染.

表 3 底泥有机氯农药含量及潜在生态风险指数”

Tab. 3 Organochlorine pesticide contents and potential ecological risk index in sediments

\begin{tabular}{|c|c|c|c|c|c|}
\hline 农药名称 & ERL(ng/g) & $\operatorname{ERM}(\mathrm{ng} / \mathrm{g})$ & 东太湖 (ng/g) & 湖心 $(\mathrm{ng} / \mathrm{g})$ & 五里湖 (ng/g) \\
\hline$\alpha-\mathrm{HCH}$ & - & - & nd & nd & 0.51 \\
\hline$\beta-\mathrm{HCH}$ & - & - & nd & nd & 0.45 \\
\hline$\gamma$-HCH & - & - & nd & nd & 0.47 \\
\hline$\Sigma(\mathrm{HCH})$ & - & 100 & nd & nd & 1.43 \\
\hline 4. 4' -DDE & 6.75 & 50 & 0.75 & 0.91 & 1.24 \\
\hline Atrazine & - & - & nd & nd & nd \\
\hline Simazine & - & - & nd & nd & nd \\
\hline Methyl Parathion & - & - & nd & nd & nd \\
\hline Aldrin & - & 40 & nd & nd & nd \\
\hline Endrin & 62.4 & 500 & nd & nd & nd \\
\hline Heptachlor & - & 10 & nd & nd & nd \\
\hline Disulfoton & - & - & nd & nd & nd \\
\hline Sulfotep & - & - & nd & nd & nd \\
\hline
\end{tabular}


表 4 底泥堆场原土壤和堆场使用 8 个月后 PAHs 含量及潜在生态风险*

Tab. 4 Potential ecological risk of PAHs in original soil in the stockyard and 8 months after uses

\begin{tabular}{|c|c|c|c|c|}
\hline PAHs & ERL( ng/g) & $\operatorname{ERM}(\mathrm{ng} / \mathrm{g})$ & 堆场底泥 (使用 8 个月后) & 堆场原土壤 \\
\hline 䒺 & 160 & 2100 & 14.72 & 4.41 \\
\hline 芴 & 19 & 540 & 3.00 & 4.17 \\
\hline 菲 & 240 & 1500 & 3.47 & 0.87 \\
\hline 蒽 & 85 & 1100 & 17.80 & 3.26 \\
\hline 苂葸 & 600 & 5100 & 34.42 & 6.84 \\
\hline 芘 & 665 & 2600 & 32.63 & 4.05 \\
\hline 䓛 & 384 & 2800 & nd & 23.73 \\
\hline 苯并 (a)葸 & 261 & 1600 & 8.85 & 0.65 \\
\hline 苯并 (a) 芘 & 430 & 1600 & nd & nd \\
\hline 苯并 $[\mathrm{B}$ ]苂葸 & 520 & 1600 & 126.77 & nd \\
\hline SUM & 4022 & 44792 & 241.7 & 48.0 \\
\hline
\end{tabular}

* nd 表示未检出.

由于这些植物原先生长的堆场土壤中 PAHs 含量较低, 仅为五里湖疏竣底泥中含量的约 $1 / 6$, 湖泊底泥 输人到堆场内后, 植物接受了更高的 $\mathrm{PAHs}$ 负荷,生物累积作用使水花生在堆场接受疏竣底泥 8 个月后, 秸 秆内的 PAHs 含量增加了 6 倍, 显示了强烈的累积作用. 且由于生物累积作用,植物中的部分单体多环芳烃 含量甚至高于疏竣底泥, 而植物体内的 PAHs 总含量也高于疏竣底泥,约为疏竣底泥中含量的 3.5 倍. 更证 明了生物累积作用将底泥中的多环芳烃含量得到了放大. 但目前没有进一步的证据表明这样的生物放大 作用对堆场内的水生植物有否毒害作用,将在进一步的研究中阐明.

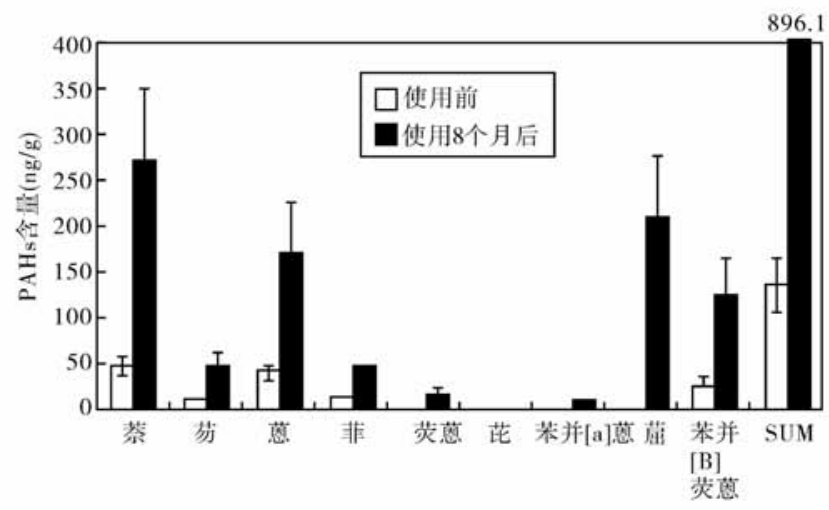

图 3 堆场使用前后水生植物 (水花生) 多环芳烃含量变化

Fig. 3 Content change of PAHs in the water plant (alligator alternanthera) before and after the use of slurry stockyard

\section{5 结论}

五里湖疏竣区底泥中的多环芳烃和有机氯农药含量高于太湖其他湖区,但与其他湖泊相比,含量中等. 若采用 Long 等人提出的沉积物环境质量基准判断, 就多环芳烃的含量而言,堆场原土壤和堆场堆放底泥后 都基本表现为生态安全,但由于生态效应的影响仅凭该环境质量基准判断仍可能导致认识偏差,因此,采用 其他的必要手段,如暴露分析等进行综合分析有助于提高判断的准确性. 由于多环芳烃类物质的保守性, 堆场在使用 8 个月后,该类物质未发生显著的累积或者降解. 由于生物累积作用,堆场使用后,其生长的植 物 (水花生) 体内多环芳烃类物质含量增加了 6 倍, 且新检测出了原来堆场使用前植物体内不含有的苯并 
[a]葱和䓛,说明堆场内的堆放五里湖疏竣底泥后对植物产生了一定的生态影响. 但疏竣后对堆场的底泥 和相应受体的毒性效应研究仍需结合其他生态风险评价手段进行.

\section{6 参考文献}

[1] National sediment quality survey, Appendix D. Screening values for Chemicals evaluated( http://www. epa. gov).

[2] Mohamed T A, Naglaa L, Youssof Y. Residues of chlorinated hydrocarbons, polycyclic aromatic hydrocarbons and polychlorinated biphenyls in some marine organisms in Lake Temsah, Suez Canal, Egypt. Aquatic Ecosystem Health \& Management, 2001,4(2):165 - 173.

[3] Kelderman PMW, Drossaert M E et al. Pollution assessment of the canal sedimentsin the city of Delft (the Netherlands). Wat Res,2000, 34(3) : 936 - 944.

[4] Long E R, Chapman P M. A sediment quality triad: Measures of sediment contamination, toxicity and infaunal community composition in Puget Sound. Marine Poll Bull,1985,16(10):405 - 415.

[5] Long E R, Morgan L G. The potential for biological effects of sediment sorbed contaminants tested in the National Status and Trends Program. NOAA tech. memo. NOA OMA 52. National Oceanic and Atmospheric Administration, Seattle, WA. ,1990.

[6] Long E R, MacDonald D D, Smith S L et al. Incidence of adverse biological effects within ranges of chemical concentrations in marine and estuarine sediments. Environ Manage,1995, 19(1) :81 - 97.

[7] Long E R, Field L J, MacDonald D D. Predicting toxicity in marine sediments with numerical sediment quality guidelines. Environmental Toxicology and Chemistry, 1998,17:714-727.

[8] http://www. noaa. gov.

[9] 张 路, 范成新, 秦伯强等. 太湖宜溧河水系沉积物中多环芳烃来源解析. 地球化学, 2003, 32(2): $124-130$.

[10] 翟文川, 王苏民, 吴瑞金等. 东太湖沉积物中多环芳烃 - 菲系列化合物的来源探讨. 湖泊科学, $2001,13(1): 9-14$. 